\title{
Sound source localization - state of the art and new inverse scheme
}

\author{
S. Gombots, J. Nowak(D, M. Kaltenbacher
}

\begin{abstract}
Acoustic source localization techniques in combination with microphone array measurements have become an important tool for noise reduction tasks. A common technique for this purpose is acoustic beamforming, which can be used to determine the source locations and source distribution. Advantages are that common algorithms such as conventional beamforming, functional beamforming or deconvolution techniques (e.g., Clean-SC) are robust and fast. In most cases, however, a simple source model is applied and the Green's function for free radiation is used as transfer function between source and microphone. Additionally, without any further signal processing, only stationary sound sources are covered. To overcome the limitation of stationary sound sources, two approaches of beamforming for rotating sound sources are presented, e.g., in an axial fan.

Regarding the restrictions concerning source model and boundary conditions, an inverse method is proposed in which the wave equation in the frequency domain (Helmholtz equation) is solved with the corresponding boundary conditions using the finite element method. The inverse scheme is based on minimizing a Tikhonov functional matching measured microphone signals with simulated ones. This method identifies the amplitude and phase information of the acoustic sources so that the prevailing sound field can be with a high degree of accuracy.
\end{abstract}

Keywords: sound source localization; inverse scheme; rotating beamforming; beamforming

\section{Schallquellenlokalisation: state of the art und neues inverses Verfahren.}

Die Lokalisation von akustischen Schallquellen mithilfe von Schalldruckmessungen unter Verwendung von Mikrofonarrays ist ein wichtiges Instrument in der Lärmbekämpfung. Ein weit verbreitetes Verfahren ist hierbei das akustische Beamforming, mit dem sowohl die Quellpositionen als auch -verteilungen bestimmt werden können. Bekannte Algorithmen, wie Standard-Beamforming, FunctionalBeamforming oder Entfaltungsmethoden (wie z. B. Clean-SC) haben den Vorteil, dass sie robust und schnell in der Berechnung sind. Nachteilig ist hingegen, dass meist ein simples Modell für die akustischen Quellen angenommen wird, und dass die Green'sche Funktion für freie Schallabstrahlung als Transferfunktion zwischen Quell- und Mikrofonpositionen verwendet wird. Außerdem können bewegte Schallquellen nicht ohne weitere Signalverarbeitungsschritte lokalisiert werden. In diesem Zusammenhang werden zwei Methoden für rotierende Schallquellen, wie sie z. B. bei einem rotierenden Ventilator vorkommen, präsentiert.

Für eine akkurate Berücksichtigung der Messumgebung und um die Einschränkungen bezüglich des vereinfachten Quellenmodells und der Randbedingungen der Messumgebung zu überwinden, wird ein inverses Verfahren präsentiert, in dem die Wellengleichung im Frequenzbereich (Helmholtz-Gleichung) mit entsprechenden Randbedingungen mittels der Finite Elemente-Methode gelöst wird. Dieses Verfahren basiert auf einem Tikhonov-Funktionals, das die Differenz zwischen Mikrofonmessungen und den simulierten Schalldrücken minimiert. Mit dieser inversen Methode können die Schallquellen in Amplitude und Phase identifiziert werden, sodass das vorherrschende Schallfeld mit hoher Genauigkeit rekonstruiert werden kann.

Schlüsselwörter: Schallquellenlokalisation; inverses Verfahren; Beamforming; rotierendes Beamforming

Received February 9, 2021, accepted March 12, 2021, published online March 25, 2021

(c) The Author(s) 2021

\section{Introduction}

Typically, sound emissions from technical applications and production machines are perceived as disturbing noise. When trying to solve noise problems or refine the acoustic design of a product, knowledge of the position and distribution of sound sources is necessary. In this context, one of the biggest challenges in noise and vibration problems is to identify the areas of a device, machine or structure that produce the significant acoustic emission. For this task various sound localization methods can be used, in order to localize and visualize sound sources. The information will be given in so-called source maps, which provide information about location, distribution and strength of sound sources.

The standard methods are intensity measurements, acoustic nearfield holography and acoustic beamforming. But, these methods are not universally applicable. In contrast to intensity measure- ments, where an intensity probe is used, near-field holography and beamforming use locally distributed microphones (= microphone array). Depending on the measurement object, frequency range and measurement environment, the different methods have specific strengths and weaknesses.

In the last years, considerable improvements have been achieved in the localization of sound sources using microphone arrays. However, there are still some limitations. In most cases, a simple source model is applied and the Green's function for free radiation is used as transfer function between source and microphone. Hence, the

Gombots, Stefan, TU Wien, Institute of Mechanics and Mechatronics, Wien, Austria (E-mail: stefan.gombots@tuwien.ac.at); Nowak, Jonathan, TU Wien, Institute of Mechanics and Mechatronics, Wien, Austria; Kaltenbacher, Manfred, TU Graz, Institute of Fundamentals and Theory in Electrical Engineering, Graz, Austria 


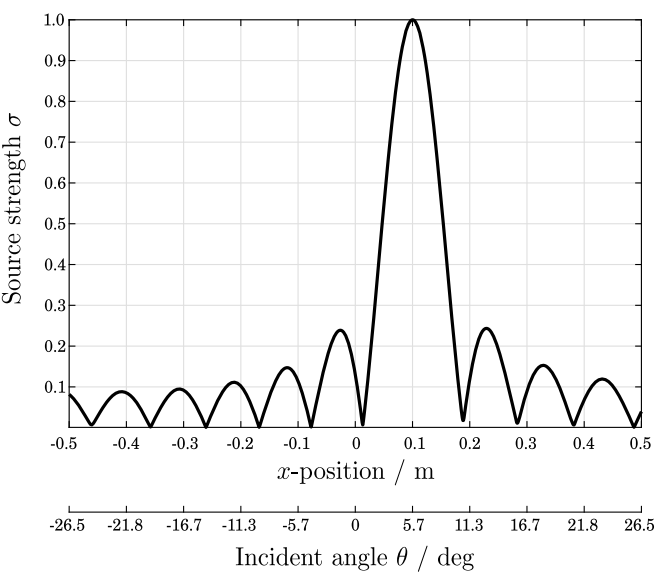

(a) Source map.

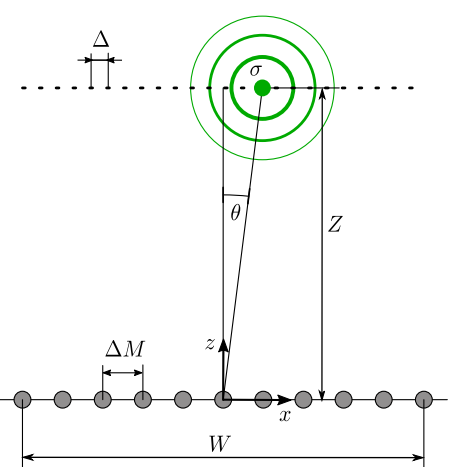

(b) Geometric setting

Fig. 1. Parameters: Distance to scanning area $Z=1 \mathrm{~m}$, array aperture $W=1 \mathrm{~m}$, source frequency $f=4000 \mathrm{~Hz}$, microphone spacing $\Delta M=0.1 \mathrm{~m}$ and discretization of the focus grid $\Delta=1 \mathrm{~mm}$

actual conditions as given in the measurement setup are not taken into account.

In real life applications, one may also be faced with moving sound sources, e.g. a passing vehicle or a rotating fan. Here, the mentioned sound source localization algorithms and methods do not readily apply, but there exist advanced signal processing methods that overcome the limitation to stationary sound sources.

\section{Beamforming based algorithm}

The acoustic field, described by the complex acoustic pressure $\widetilde{p}_{a}$ of a monopole source with source strength $\sigma$, is calculated in the frequency domain with Green's function $\widetilde{g}(r)$ of free radiation by

$$
\tilde{p}_{\mathrm{a}}(\boldsymbol{x}, \boldsymbol{y}, \omega)=\sigma(\boldsymbol{y}) \tilde{g}(r)=\sigma(\boldsymbol{y}) \frac{\mathrm{e}^{-\mathrm{j} \frac{\omega}{c_{0}} r}}{4 \pi r} \quad \text { with } \quad r=|\boldsymbol{x}-\boldsymbol{y}|,
$$

where $\boldsymbol{x}$ denotes the observer position (e.g. microphone positions) and $\boldsymbol{y}$ the source postions, $c_{0}$ the speed of sound and $\omega=2 \pi f$ the angular sound frequency. All measured microphone signals $p_{a}(t)$ are Fourier transformed and the resulting complex pressure values $\widetilde{p}_{\mathrm{a}}$ at a certain frequency $\omega$ are stored in a vector

$$
\widetilde{\boldsymbol{p}}_{\mathrm{a}}(\omega)=\left[\begin{array}{c}
\widetilde{p}_{\mathrm{a}, 1}(\omega) \\
\vdots \\
\tilde{p}_{\mathrm{a}, M}(\omega)
\end{array}\right] .
$$

The cross-spectral matrix (CSM) is calculated by

$$
\widetilde{\boldsymbol{C}}(\omega)=\widetilde{\boldsymbol{p}}_{\mathrm{a}}(\omega) \widetilde{\boldsymbol{p}}_{\mathrm{a}}^{\mathrm{H}}(\omega),
$$

with $\square^{\mathrm{H}}$ the hermitian operation (transposition and complex conjugation).

In Conventional Beamforming (ConvBF), the fundamental and most basic as well as robust frequency domain processing method [1], the measured sound field is compared to a calculated sound field. Thereby, a certain model for the acoustic source is assumed. Most beamforming algorithms model the acoustic source by monopols (1) to calculate the acoustic pressure. By using this acoustic source model, following functional is defined

$$
\mathcal{J}\left(\sigma^{2}\right)=\left\|\widetilde{\boldsymbol{C}}-\sigma^{2} \widetilde{\boldsymbol{g}} \widetilde{\boldsymbol{g}}^{H}\right\|_{F}^{2},
$$

which refers to a single source with the strength $\sigma$ to be determined (without loss of generality). Here, $\widetilde{\boldsymbol{g}}$ denotes the vector of the individual Green's functions (also called steering vector) and $\|\square\|_{F}$ is the Frobenius norm. Minimizing the functional (3), i.e. setting its derivative to zero, yields

$$
\sigma(\boldsymbol{y})=\sqrt{\frac{\widetilde{\boldsymbol{g}}^{\mathrm{H}} \tilde{\boldsymbol{c}} \widetilde{\boldsymbol{g}}}{\left(\widetilde{\boldsymbol{g}}^{\mathrm{H}} \widetilde{\boldsymbol{g}}\right)^{2}}}=\sqrt{\widetilde{\boldsymbol{w}}^{\mathrm{H}} \widetilde{\boldsymbol{C}} \widetilde{\boldsymbol{w}}} \quad \text { with } \quad \widetilde{\boldsymbol{w}}=\frac{\widetilde{\boldsymbol{g}}}{\widetilde{\boldsymbol{g}}^{\mathrm{H}} \widetilde{\boldsymbol{g}}^{\prime}}
$$

which is the expression for the determination of the source strength. Thereby, $\widetilde{\boldsymbol{w}}$ denotes the weighted steering vector. The steering vector represents the transfer functions from the focus point to the microphone positions and account for the phase shift and amplitude correction (sound propagation model) as well as the microphone weighting [2]. They can be either obtained by measurements [3] or by theoretical models. In [4], different steering vector formulations are discussed. Thereby, a reasonable enhancement in the correct estimation of the source location could be obtained, whereas there is a trade-off between the correct reconstruction of the location and the source strength. Another approach of determining the transfer function is given by combining measurement and simulation, leading to numerically calculated transfer functions (NCTFs) $[5,6]$.

The main diagonal elements of the CSM (2) represent the auto power of the microphones and therefore provide no information about the phase differences between the microphones, but may introduce microphone self noise. Hence, for experimental measurements, the main diagonal is usually omitted.

For broadband sound sources it may be of interest to plot source maps in frequency bands (e.g., one-third or octave bands), rather than for single frequencies. For this purpose the individual source maps are energy summed [7] for each frequency band according to

$$
\sigma_{n}=\sqrt{\sum_{i=1}^{N_{f}} \sigma_{n, i^{\prime}}^{2}}
$$

whereby $N_{f}$ denotes the number of frequencies in the considered band.

In Fig. 1a the source map (calculated by (4)) of the geometric setup given in Fig. $1 \mathrm{~b}$ is depicted. Here, for the localization a line 


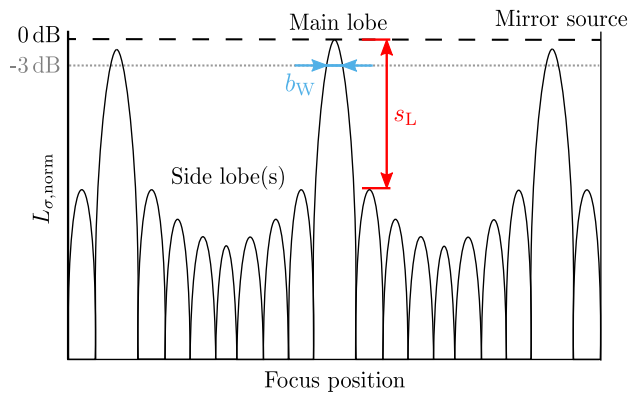

(a) Line array.

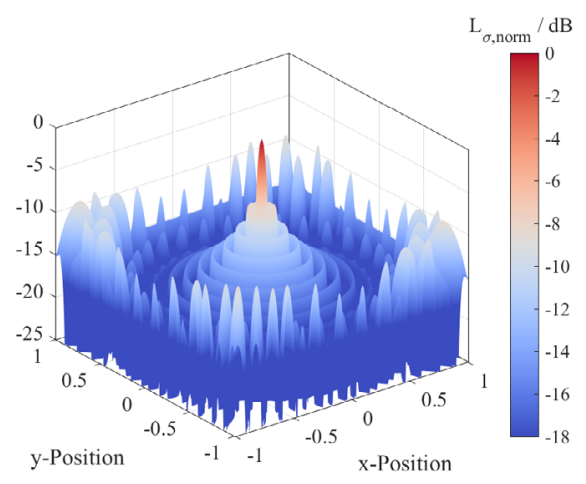

(b) Ring array.

Fig. 2. Schematic representation of a Point Spread Function (PSF) in (a) for a line array (1D) and in (b) for a ring array (2D)

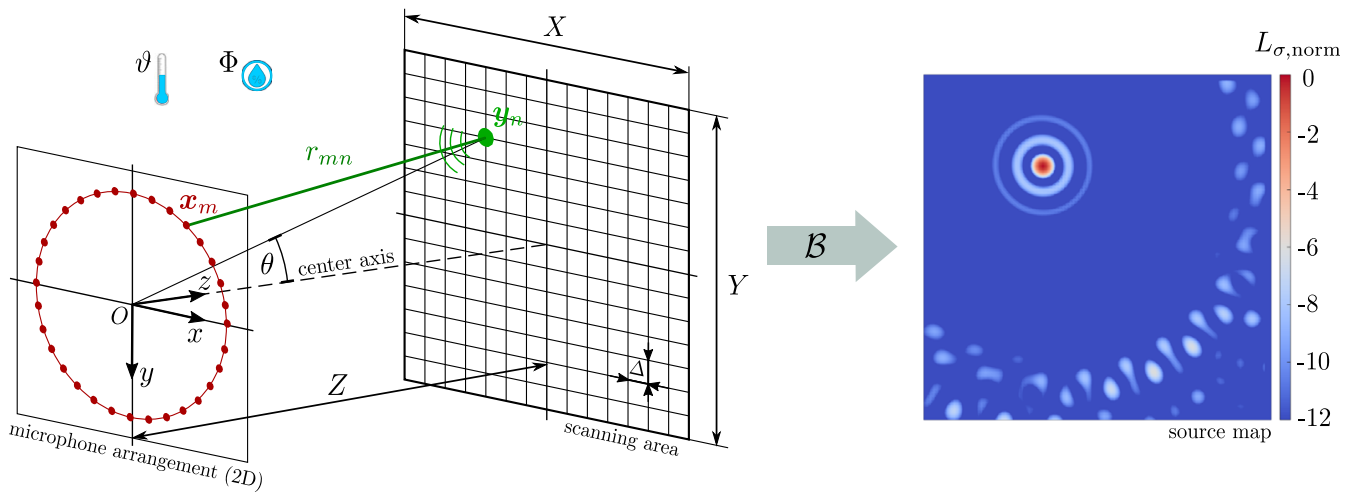

Fig. 3. Overview of the parameters for acoustic beamforming when using a two-dimensional array including the obtained source map after the beamforming process

array was applied. In the source map, the actual source position is clearly visible through the maximum which is also called main lobe. The source strength will be given as source level

$$
L_{\sigma}=20 \log \frac{\sigma}{\sigma_{\text {ref }}},
$$

with the reference value $\sigma_{\text {ref }}=20 \mu \mathrm{Pam}^{d-2}$ ( $d$ is the space dimension), according to the reference value of the sound pressure level. Another important quantity is the normalized source level

$$
L_{\sigma, \text { norm }}=20 \log \frac{\sigma}{\sigma_{\max }},
$$

where $\sigma_{\max }$ represents the maximum source strength in the considered focus grid.

\subsection{Beamforming parameters}

The source map, Eq. (4), can be interpreted as a convolution of the array response function (= point spread function, PSF) with the real source distribution [2]. Thus, the main lobe is surrounded by socalled side lobes (artefacts) (see Fig. 2) whereby the formation of them is purely beamforming-related and independent of measuring equipment. Hence, the limitation of resolution and dynamic is caused by the PSF of the microphone array. The PSF has a strong and wide main lobe as well as strong side lobes especially for low frequencies, such that weaker sources may be hidden. The side lobes may lead to problems in the localization and interpretation of the source map if several sources are present. If weak sources have to be localized in the presence of strong sources, the distance between main and side lobes $s_{L}$ should be as large as possible (see Fig. 2a). The PSF depends among others on

- the source characteristics (frequency, position, strength),

- the spatial arrangement of the microphones,

- the number of microphones and

- the focus grid.

Hence, the layout of the microphone array (spatial arrangement, microphone number) is crucial for the quality of the source map and for the ability to locate and quantify acoustic sources. To assess the capabilities, the response function of the array (= PSF) to a defined sound source (usually a point source) can be used. With the PSF, statements can be made about the properties for individual frequencies on the current setup (focus grid, microphone arrangement, etc.). This makes it possible to design microphone arrays for specific measurement tasks or to test the performance of an existing arrangement. To assess the PSF, the beam width $b_{W}$ and the side lobe attenuation $s_{L}$ is used. The width of the main lobe $b_{W}$ (usually specified $3 \mathrm{~dB}$ below the maximum, see Fig. 2a) limits the spatial resolution. Thereby, the theoretical resolution is given by the Rayleigh limit [8] as well as by the Sparrow limit [9].

Next, all important parameters that are necessary and must be considered for acoustic beamforming are summarized (see Fig. 3): 
- Array layout (microphone number $M$, microphone spacing $\Delta M$, aperture $W$ ),

- Focus (scan) grid (discretization $\Delta$, dimension $X$ and $Y$ ),

- Distance between microphone array and focus grid $Z$,

- Temperature $\theta$ and relative air humidity $\Phi$ for the estimation of $c_{0}$,

- Beamforming algorithm $\mathcal{B}$

- Steering vector $\widetilde{\boldsymbol{w}}$ formulation.

\subsection{Functional beamforming}

One drawback of ConvBF is that the source map obtained with Con$\mathrm{VBF}$ not only contains the main lobe, i.e. the peak in the source map where the actual source is located, but also shows artefacts (side lobes), which occur at positions without actual sources. This is due to the above mentioned fact that the computed source distribution is a convolution of the real source distribution with the particular response function (PSF) of the array. While these side lobes have a smaller amplitude than the main lobe it is still possible that the side lobes obscure weaker sources which are therefore not detected by ConvBF. One possibility to reduce the high side lobe level is to use an advanced beamforming algorithm called Functional beamforming (FuncBF) $[10,11]$. Here, a parameter $v$ is introduced and (4) is changed to

$$
\sigma=\sqrt{\frac{\left(\widetilde{\boldsymbol{g}}^{H} \widetilde{\boldsymbol{C}}^{\frac{1}{v}} \tilde{\boldsymbol{g}}\right)^{v}}{\left(\widetilde{\boldsymbol{g}}^{\mathrm{H}} \tilde{\boldsymbol{g}}\right)^{v+1}} .}
$$

The CSM is diagonalised according to

$$
\widetilde{\boldsymbol{C}}=\tilde{\boldsymbol{U}}_{\Lambda} \tilde{\boldsymbol{U}}^{\mathrm{H}}
$$

with $\boldsymbol{\Lambda}=\operatorname{diag}\left(\lambda_{1}^{\frac{1}{v}}, \ldots, \lambda_{M}^{\frac{1}{v}}\right)$ consisting of the eigenvalues $\lambda_{i}$ of $\widetilde{\mathbf{C}}$.

The case of $v=1$ leads to the equation for ConvBF. Using FuncBF, the dynamic range and the resolution of the source map can be improved for increasing values of the parameter $v \geq 1$. In practice, using well calibrated arrays values of up to $v=100$ leads to satisfying results [10].

\subsection{Deconvolution algorithms}

Another way to overcome the drawback of side lobes in the source map is to use advanced signal processing algorithms based on deconvolution, e.g., DAMAS [12], Clean-SC [13], SC-DAMAS [14] etc., that convert the raw source map (4) into a deconvoluted source map, resulting in higher resolution and dynamic range. Thereby, it is assumed that the computed source map obtained by (4) is built up by individual scaled PSFs of the array. By using deconvolution algorithms, these response functions of the array are determined and replaced by single peaks or narrow-width beams. As a consequence, the side lobes (artefacts) are removed in the deconvolved map. In [15] and [16], one can find a detailed comparison between different deconvolution techniques and the application to $2 \mathrm{D}$ and $3 \mathrm{D}$ sound source localization. There, the different techniques are compared with respect to position detection, source level estimation and computational time. The main findings for source localization in a free radiation environment can be summarized as follows: (1) SC-DAMAS provides the best source map at highest computational costs; (2) Clean-SC has the best trade-off between fast computation and correct source detection. Usually, the deconvolution algorithms can only be applied in post-processing because the calculation of deconvolved source maps takes too much time for real-time analyses.

\subsection{Rotating beamforming}

If the sound source is moving, i.e. the distance between sound source and microphone (observer) is time dependent $r=r(t)$ one has to take into account that sound which is received at time $t=$ reception time) at an observation point $\boldsymbol{x}$ was emitted at an earlier time $\tau$ (= retarded time or emission time) from the source point $\boldsymbol{y}$. Further, a stationary observer perceives different sound frequencies than those that are emitted by the source, which is commonly known as the Doppler effect.

The retarded time is defined implicitly by

$$
c_{0}(t-\tau)-r(\tau)=0
$$

For moving sound sources the presented methods for sound source localization can no longer be applied but there exist signal processing algorithms that treat special cases, e.g. translationally moving sound sources at constant speed $U$ or sound sources rotating at an angular velocity $\Omega$.

If we assume a time dependent monopole that moves along a path $\boldsymbol{x}=\boldsymbol{x}_{\mathbf{S}}(t)$ the inhomogeneous wave equation in time domain reads as

$$
\frac{1}{c_{0}^{2}} \frac{\partial^{2} p_{a}}{\partial t^{2}}-\Delta p_{a}=q(\boldsymbol{x}, t)=Q(t) \delta\left(\boldsymbol{x}-\boldsymbol{x}_{\mathbf{s}}(t)\right) .
$$

Its solution, i.e. the acoustic pressure of a moving monopole, can be calculated as [17]

$$
p_{\mathrm{a}}(\boldsymbol{x}, t)=\frac{Q_{\mathrm{e}}}{4 \pi r_{\mathrm{e}}\left(1-M_{\mathrm{e}} \cos \vartheta_{\mathrm{e}}\right)}
$$

with

$$
\begin{aligned}
\cos \vartheta & =\frac{\boldsymbol{r} \cdot \boldsymbol{M}}{r M} \\
\boldsymbol{r}(\tau) & =\boldsymbol{x}-\boldsymbol{x}_{\mathrm{s}}(\tau) ; \quad r=|\boldsymbol{r}| \\
\boldsymbol{M} & =\left.\frac{1}{c_{0}} \frac{\partial \boldsymbol{x}_{\mathrm{S}}}{\partial t}\right|_{\tau} ; \quad M=|\boldsymbol{M}|,
\end{aligned}
$$

where $\square_{\mathrm{e}}$ denotes evaluation at retarded time $\tau, \boldsymbol{M}$ denotes the vectorial Mach number and $\vartheta$ the angle between the vector of the source velocity $c_{0} \boldsymbol{M}$ and the vector between source and observer $\boldsymbol{r}(\tau)$.

In the stationary case the sound pressure emitted by a monopole source at location $\boldsymbol{y}$ is given as

$$
p_{a}(\boldsymbol{x}, t)=\frac{Q\left(t-|\boldsymbol{x}-\boldsymbol{y}| / c_{0}\right)}{4 \pi r} .
$$

The factor $1 /\left(1-M_{\mathrm{e}} \cos \vartheta_{\mathrm{e}}\right)$ in the non-stationary case is also called 'Doppler factor' as the momentarily perceived sound frequency $f(t)$ at the observer point of a sound source that emits sound at constant frequency $f_{0}$ calculates as

$$
f(t)=\frac{d}{d t}\left(f_{0} \tau\right)=\frac{f_{0}}{1-M_{\mathrm{e}} \cos \vartheta_{\mathrm{e}}} .
$$

Since rotating sound sources often occur in practise, e.g. rotating fans, we shall take a closer look at the signal processing algorithms for these kind of sound sources. The sound field that is generated by a rotating monopole with sound frequency $f_{0}=1500 \mathrm{~Hz}$, evaluated at a single observation point, is depicted in Fig. 4. In the frequency domain (Fig. 4b) one can see that the sound field at the observation point not only consists of the excitation frequency $f_{0}$ but additionally of values at frequencies shifted by $m \Omega /(2 \pi)$. 


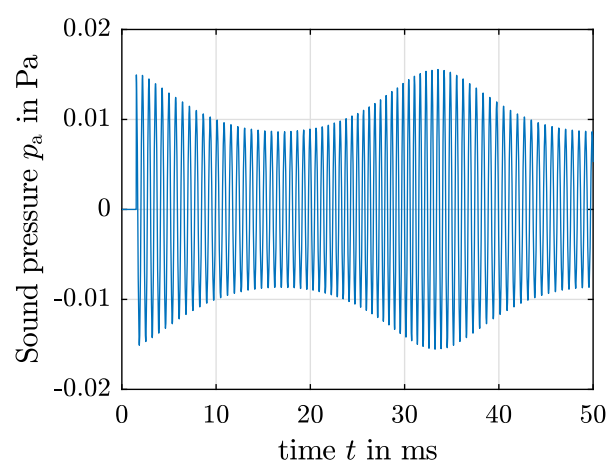

(a) Time domain.

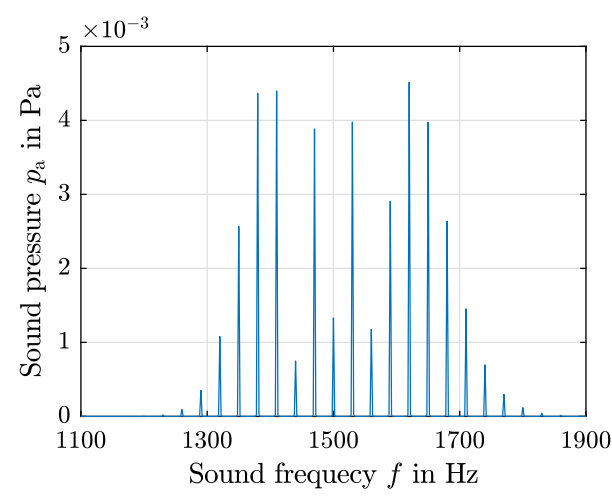

(b) Frequency domain.

Fig. 4. Sound pressure generated by a rotating monopole, sound frequency $f_{0}=1500 \mathrm{~Hz}$, rotating at $\Omega=2 \pi 30 \mathrm{~s}^{-1},\left.x_{\mathrm{s}}\right|_{\tau=0}=[0.3,0,0.5] \mathrm{m}$, observer position $x=[0.45,0,0] \mathrm{m}$

We will focus on two methods that make sound source localization with rotating sources possible, the interpolation of the pressure signals in the time domain $[18,19]$ and the spinning mode decomposition in the frequency domain e.g. [18]. Both methods requires equally spaced microphones on a ring array and a common axis of the ring array and the source's rotational axis.

In the former method the sound pressure signals recorded by the stationary microphones are interpolated according to the momentary position of the rotating sound source. This leads to virtually rotating acoustic pressure signals $p_{\mathrm{vr}, m}$ at microphone $m$ which is calculated from its neighboring microphones $m_{l}$ and $m_{\mathrm{h}}$ as

$$
p_{\mathrm{vr}, m}(t)=s_{\mathrm{I}} p_{m_{1}}+s_{\mathrm{h}} p_{m_{\mathrm{h}}},
$$

with

$$
\begin{aligned}
s_{\mid}(t) & =\frac{\varphi(t)}{\Delta \varphi}-\left\lfloor\frac{\varphi(t)}{\Delta \varphi}\right\rfloor, \\
s_{\mathrm{h}}(t) & =1-s_{\mid}(t) .
\end{aligned}
$$

Here, $\varphi(t)$ denotes the angle of the source, $\Delta \varphi$ is the angle between two arbitrary neighbouring microphones and $\lfloor\square\rfloor$ denotes the floor function.

The latter method for compensating the rotation of a sound source uses modal decomposition of the acoustic pressure signals in the frequency domain and a modified Green's function for the rotating monopole as steering vectors [18]. This method is, contrary to the interpolation method, analytically exact. It requires a constant rotational frequency of the source $\Omega=$ const. A measured microphone signal $\widetilde{p}_{m}(\omega)$ at microphone $m$ is expressed via a discrete Fourier series with spinning mode coefficients

$$
\widetilde{p}_{n}(\omega)=\frac{1}{M} \sum_{m=1}^{M} \widetilde{p}_{m}(\omega) \mathrm{e}^{-\mathrm{j} n \varphi_{m}},
$$

with

$$
-\frac{M}{2}+1 \leq n \leq \frac{M}{2} .
$$

The pressure signals in the rotating reference frame $\widetilde{p}_{\Omega}$ are then calculated as

$$
\tilde{p}_{\Omega}\left(\varphi_{m}, \omega\right)=\sum_{n=-M / 2+1}^{M / 2} p_{n}(\omega+n \Omega) \mathrm{e}^{\mathrm{j} n \varphi_{m}} .
$$

With the modified pressure signals $\widetilde{\boldsymbol{p}}_{\mathrm{vr}}$ and $\widetilde{\boldsymbol{p}}_{\Omega}$, respectively, one can then calculate a modified CSM analogously to (2) and beamforming maps can be calculated in the same way as in the stationary case.

When using the modified CSM but stationary steering vectors, one only gets approximate source locations. For rotating sources the source maps are shifted in tangential direction. This can be avoided when using the modified Green's function $g_{\Omega}$ or corrected distances $\boldsymbol{r}^{*}$ between each scan point and each microphone that take into account that the sound source has moved from its initial position when sound is received at a specific microphone - see (10). Further details on the modified steering vectors can be found in $[18,20,21]$.

\subsection{Limitations and challenges}

The fundamental processing method, ConvBF also called frequency domain beamforming (FDBF) [1], is robust and fast. In this simple method, limitations regarding resolution and dynamic range are caused by the PSF. A modification of this classic approach is delivered by FuncBF (Sect. 2.2), which leads to an improvement of resolution and dynamic range, whereby the computational cost remains almost the same as in the standard approach. Furthermore, deconvolution techniques (Sect. 2.3) can be applied, which attempt to eliminate the influence of the PSF on the raw source map. Thereby, resolution and dynamic range are greatly improved at the costs of computational time.

Despite these advances in beamforming techniques, it has to be mentioned that major limitations are caused by the source model. Most beamforming algorithms model the acoustic sources as monopoles or/and dipoles. Moreover, the steering vector $\widetilde{\boldsymbol{g}}$, describing the transfer function (TF) between source and microphone, is modeled by Green's function for free radiation. A different choice of steering vectors can improve the results as demonstrated in [4]. A further challenge of these methods are source localization at low frequencies and in environments with partially or fully reflecting surfaces, for which beamforming techniques do not provide physically reasonable source maps. Furthermore, obstacles can not be considered. In such cases, the steering vectors have to be adapted to take the reverberant environment into account. Two approaches are considered in [3]: (1) modeling reflections by a set of monopoles located at the image source positions; (2) experimentally based identification of Green's function. Thereby, the best results could be obtained by using the formulation with the experimentally obtained Green's functions. 
Beamforming is mainly used for acoustic source localization rather than for obtaining quantitative source information. The qualitative statements given by the source map provides information about the distribution and position, and a relative comparison of the source strength at the considered focus grid. In many cases, this information may already be sufficient to determine the origin of the sound emission. However, sometimes quantitative source information is also needed. The estimation of quantitative source spectra is not straightforward [22], but can be obtained through integration methods. Thereby, the source map is integrated over a certain region to obtain a pressure spectrum for this specific area. Thus, the source map is required before integration. A distinction must be made between integrating the raw and deconvolved map. The deconvolved maps can be seen as ideal images of the source contribution and therefore, the integration may be done without further processing [23]. If this is done with the raw source map, it needs to be taken into account that the integrated spectra are still convolved with the array PSF. In [24], an overview of different integration methods is given.

\section{Inverse scheme}

Source localization on the basis of beamforming, can be carried out very efficiently in its simplest implementation. In literature, many different comparisons of beamforming methods can be found. Exemplarily, in [25], [2] and [26], simulated data was used as input, and in $[15,27]$, data coming from experiments. A comprehensive overview of different acoustic imaging methods can be found in [25, 28, 29]. There exist beamforming independent inverse methods (like e.g. L1Generalized Inverse Beamforming [30], Cross-spectral matrix fitting [14], etc.), which aim to solve an inverse problem considering the presence of all acoustic sources at once in the localization process. Thereby, resolution and dynamic range are greatly improved by these advanced methods at the costs of higher computational time and power.

In the provided inverse scheme a cost functional is minimized such that the physical model with source terms is fulfilled. It is based on the solution of the wave equation in the frequency domain (Helmholtz equation), which allows to fully consider realistic geometry and boundary condition scenarios. Another advantage is its easy generalizability to situations with convection and/or attenuation.

\subsection{Physical and mathematical model}

Assuming that the original geometry of the setup including the boundary conditions and the Fourier-transformed acoustic pressure signals $\widetilde{p}_{m}^{m s}(\omega)$ ( $\omega$ being the angular sound frequency, $\left.m=1, \ldots, M\right)$ at the microphone positions $\boldsymbol{x}_{m}$ are given, the physical model is represented by the Helmholtz equation. Here, we consider the following generalized form of the Helmholtz equation in the computation domain $\Omega=\Omega_{\text {acou }} \cup \Omega_{\text {damp }}$

$$
\nabla \cdot \frac{1}{\varrho} \nabla \widetilde{p}+\frac{\omega^{2}}{K} \widetilde{p}=\tilde{\sigma}^{\text {in }} \text { in } \Omega .
$$

In (24) $\tilde{\sigma}^{\text {in }}$ denotes the acoustic sources and

$$
\begin{array}{r}
\varrho(\boldsymbol{x})= \begin{cases}\tilde{\varrho}_{\text {eff }} & \text { in } \Omega_{\text {damp }} \\
\varrho_{0} & \text { in } \Omega_{\text {air }}\end{cases} \\
K(\boldsymbol{x})= \begin{cases}\tilde{K}_{\text {eff }} & \text { in } \Omega_{\text {damp }} \\
c_{0}^{2} \varrho_{0} & \text { in } \Omega_{\text {acou }}\end{cases}
\end{array}
$$

the space dependent density and compression modulus with speed of sound $c_{0}$ and mean density $\rho_{0}$. Herewith, poroelastic materials (e.g., porous absorbers) can be considered as a layer of an equivalent complex fluid having a frequency-dependent effective density $\tilde{\varrho}_{\text {eff }}$ and bulk modulus $\tilde{K}_{\text {eff }}$. With this formulation the absorption properties of surfaces can be adjusted. Thereby, a large number of models for characterization have been established for poroelastic materials. Depending on the theoretical assumptions, the models are based on a different number of (material) parameters. An overview of different modeling approaches can be found in [31]. Furthermore, the sound sources on the surface are modeled by

$$
\boldsymbol{n} \cdot \nabla \widetilde{p}=-\widetilde{\sigma}^{\mathrm{bd}} \quad \text { on } \quad \Gamma_{\text {src }}
$$

Since the identification is done separately for each frequency $\omega$, the dependence on $\omega$ is neglected in the notation. Now, the considered inverse problem is to reconstruct $\widetilde{\sigma}^{\text {in }}$ and/or $\widetilde{\sigma}^{\text {bd }}$ from pressure measurements

$$
\widetilde{p}_{m}^{m s}=\widetilde{p}\left(\boldsymbol{x}_{m}\right), \quad m=1, \ldots, M
$$

at the microphone positions $\boldsymbol{x}_{1}, \ldots, \boldsymbol{x}_{M}$. For the acoustic sources the following ansatz is made

$$
\widetilde{\sigma}^{\text {in }}+\widetilde{\sigma}^{\mathrm{bd}}=\sum_{n=1}^{N} a_{n} \mathrm{e}^{\mathrm{j} \varphi_{n}} \delta_{\boldsymbol{x}_{n}}
$$

with the searched for amplitudes $a_{1}, a_{2}, \ldots, a_{N} \in \mathbb{R}$ and phases $\varphi_{1}, \varphi_{2}, \ldots, \varphi_{N} \in[-\pi / 2, \pi / 2]$. Here, $N$ denotes the number of possible sources and $\delta_{\boldsymbol{x}_{n}}$ the delta function at position $\boldsymbol{x}_{n}$.

\subsection{Optimization based source identification}

The source identification by means of Tikhonov regularization amounts to solving the following constrained optimization problem

$$
\begin{aligned}
& \min _{\tilde{p} \in U, a \in \mathbb{R}^{N}, \varphi \in\left[-\frac{\pi}{2}, \frac{\pi}{2}\right]^{N}} J(\widetilde{p}, a, \varphi) \\
& \text { s. t. Eq. (24) is fulfilled }
\end{aligned}
$$

where $a=\left(a_{1}, \ldots, a_{N}\right), \varphi=\left(\varphi_{1}, \ldots, \varphi_{N}\right)$ and

$$
\begin{aligned}
& J(\widetilde{p}, a, \varphi)=\frac{\Psi}{2} \sum_{m=1}^{M}\left|\widetilde{p}\left(\boldsymbol{x}_{m}\right)-\widetilde{p}_{m}^{m s}\right|^{2}+\alpha \sum_{n=1}^{N}\left|a_{n}\right|^{q} \\
& +\beta \sum_{n=1}^{N} \varphi_{n}^{2}-\rho \sum_{n=1}^{N}\left(\ln \left(\frac{\pi}{2}+\varphi_{n}\right)+\ln \left(\frac{\pi}{2}-\varphi_{n}\right)\right) .
\end{aligned}
$$

In here, the box constraints on the phases $\varphi_{n}$ are realized by a barrier term with some penalty parameter $\rho>0$. This also helps to avoid phase wrapping artefacts. The penalty parameter $\rho$ and the regularization parameters $\alpha$ and $\beta$ are chosen according to the sequential discrepancy principle [32]

$$
\rho=\frac{\rho_{0}}{2^{x}}, \alpha=\frac{\alpha_{0}}{2^{x}}, \quad \beta=\frac{\beta_{0}}{2^{x}}
$$

with $x$ the smallest exponent such that following inequality

$$
\sqrt{\sum_{m=1}^{M}\left(\widetilde{p}\left(\boldsymbol{x}_{m}\right)-\widetilde{p}_{m}^{m s}\right)^{2}} \leq \varepsilon
$$

is fulfilled, with $\varepsilon$ being the measurement error. According to [33], it can be expected that this leads to a convergent regularization method. 


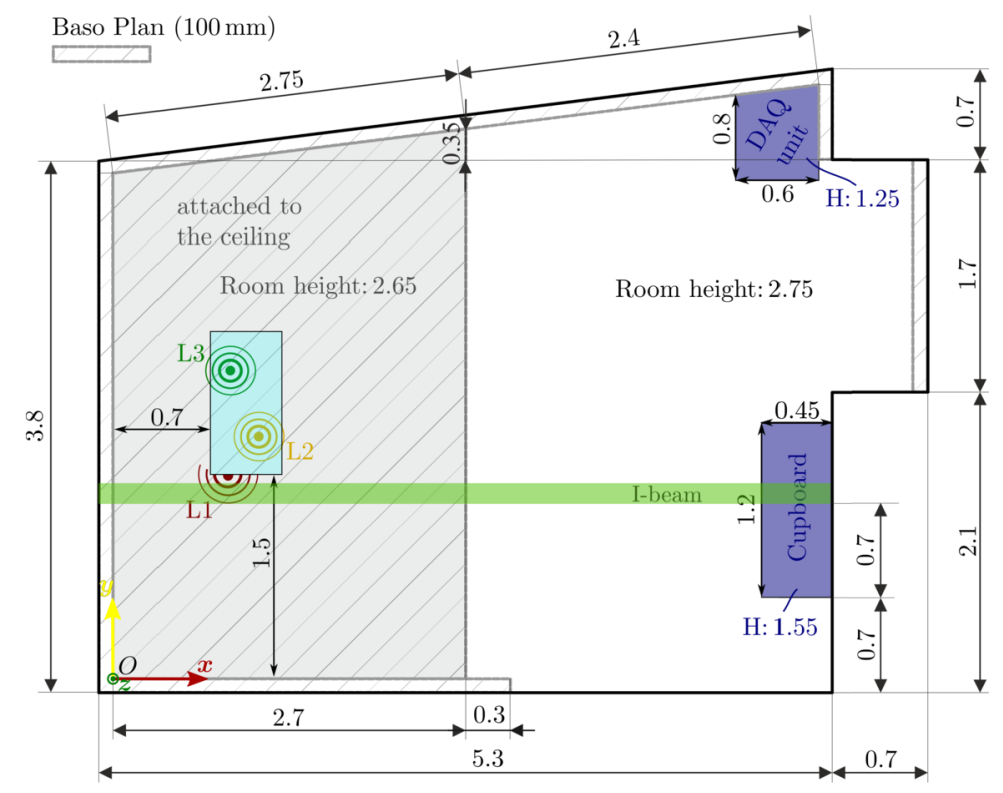

Fig. 5. Ground plan of the room with source location (dimensions in $\mathrm{m}$ )

Sparsity of the reconstruction is desired to pick the few true source locations from a large number of the $N$ trial sources. By choosing $q \in(1,2]$ close to one, an enhanced sparsity can be obtained. Since the optimization scheme uses different stopping criteria, a scaling factor

$$
\Psi=\frac{\psi}{\max \left(\left|\widetilde{p}_{m}^{m s}\right|\right)}
$$

is introduced in (29), where the maximum absolute value of the measured pressure $\left|\widetilde{p}_{m}^{m s}\right|$ is scaled to an amplitude of $\psi$.

The implemented optimization based identification algorithm is based on a gradient method with Armijo line search exploring the adjoint method to efficiently obtain the gradient of the objective function. Hence, the computational time does not depend on the number of microphones $M$ nor on the assumed number of possible sources $N$. Further details, about the inverse scheme can be found in [34]. In the current implementation, the finite element (FE) method is applied for solving the Helmholtz equation (24). Hence, the applicability of the inverse scheme towards computational time is mainly restricted to the low frequency range, since the discretization effort and therefore the number of degree of freedoms in 3D is of the order $O\left(e_{\text {size }}^{-3}\right)$, where $e_{\text {size }}$ is the mesh size being determined by

$$
\mathrm{e}_{\mathrm{size}} \approx \frac{\lambda_{\min }}{N_{\mathrm{e}}}=\frac{\mathrm{c}_{0}}{N_{\mathrm{e}} f_{\max }} .
$$

In (32) $f_{\max }$ denotes the highest frequency of the acoustic sources and $N_{\mathrm{e}}$ should be between 10 to 20 (rule of thumb [35]). Please note that any other numerical method, e.g., the boundary element method (BEM) can be applied, which may be even more efficient with respect to computation time, depending on the particular scenario.

\section{Application and results}

\subsection{Low-frequency sound source in a room}

To demonstrate the applicability of the inverse scheme in real-world scenarios, microphone array measurements were performed in a room where a generic sound source was located. Thereby, the room is partially lined with porous absorbers (Baso Plan 100) on the walls and ceiling. A ground plan of the room is shown in Fig. 5 including the generic sound source location. This sound source is a box (dimensions: $50 \times 100 \times 55 \mathrm{~cm}$ ) made of Doka formwork sheet, see Fig. 6a. The sound can be generated by three separately excitable loudspeakers (VISATON WS $20 \mathrm{E}, \varnothing=8^{\prime \prime}$ ). In order to characterize the generic source, the normal velocity is measured with a laser scanning vibrometer (LSV) Polytec PSV-500. In the measurement, first speaker L1 and afterwards L2 was active. The excitation frequency of the speakers was $250 \mathrm{~Hz}$. The measured normal velocity level $L_{\mathrm{V}}$ (ref. $50 \mathrm{~nm} / \mathrm{s}$ ) is given in Fig. $6 \mathrm{~b}$ and Fig. $6 c$ (just the side with the active speaker is shown).

The application of numerical methods like the introduced inverse scheme needs physical and geometrical modeling of the real-world situation. Therefore, first, an appropriate FE model of the measurement environment was created, which is depicted in Fig. 7. In order to obtain accurate data for an acoustic field by simulation, the boundary conditions necessary for the solution of the acoustic wave equation have to be determined in a suitable way. For the characterization of the materials present in the room, the absorption coefficient $\alpha$ was determined with impedance tube measurements applying the 2p-method (ISO 10534-2 [36]). The measured absorption coefficients showed that most of the surfaces could be assumed to be fully reflective. Hence, for these surfaces the homogeneous Neumann boundary condition is used

$$
\begin{array}{r}
\nabla \tilde{p} \cdot \boldsymbol{n}=0 \quad \text { on } \quad \partial \Omega \cup \Gamma_{\text {plaster }} \cup \Gamma_{\text {src }} \cup \Gamma_{\text {floor }} \\
\cup \Gamma_{\text {cb }} \cup \Gamma_{\text {daq }} \cup \Gamma_{\text {lbeam }} .
\end{array}
$$

For the modeling of the porous absorber an equivalent fluid model (assuming isotropic and volume averaged features) is used, which provides the effective parameters $\tilde{\varrho}_{\text {eff }}$ and $\tilde{K}_{\text {eff }}$ for the generalized Helmholtz equation (24). Hereby, the Delany-Bazely-Miki (DBM) $[37,38]$ model was chosen, which is purely empirical and derived from measurements on many highly porous materials. In Fig. 8, the fitted absorption curve of the DBM model is compared with the measurements. 


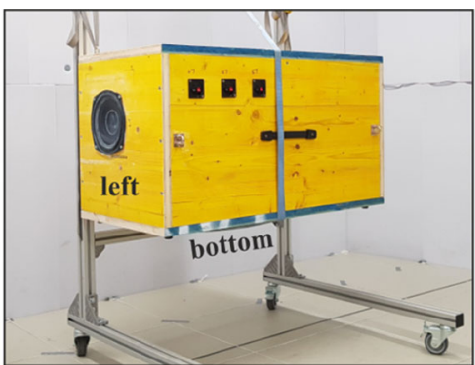

(a)

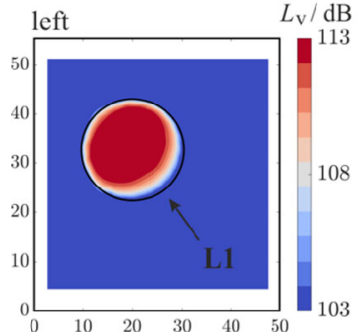

(b)

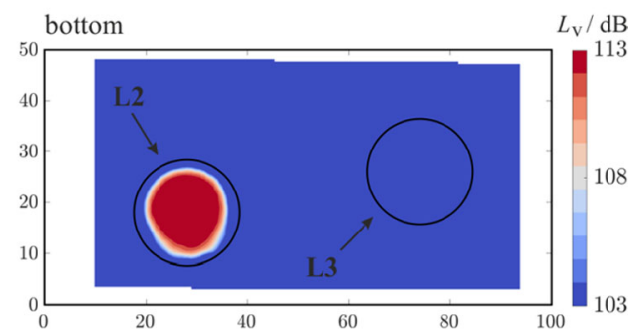

(c)

Fig. 6. (a) Generic sound source including the mounting frame. Laser scanning vibrometer measurement results given as normal velocity level $L_{v}$ (ref. $50 \mathrm{~nm} / \mathrm{s}$ ): (b) speaker L1 active, (c) speaker L2 active. Excitation frequency of the speakers: $250 \mathrm{~Hz}$

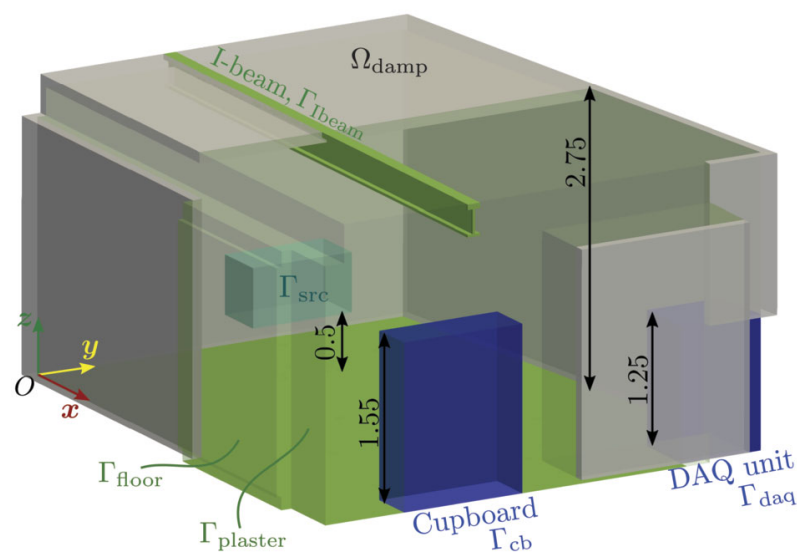

Fig. 7. Illustration of the used FE model including the labels of the regions and boundaries (dimensions in $\mathrm{m}$ ). The corresponding groundplan can be found in Fig. 5

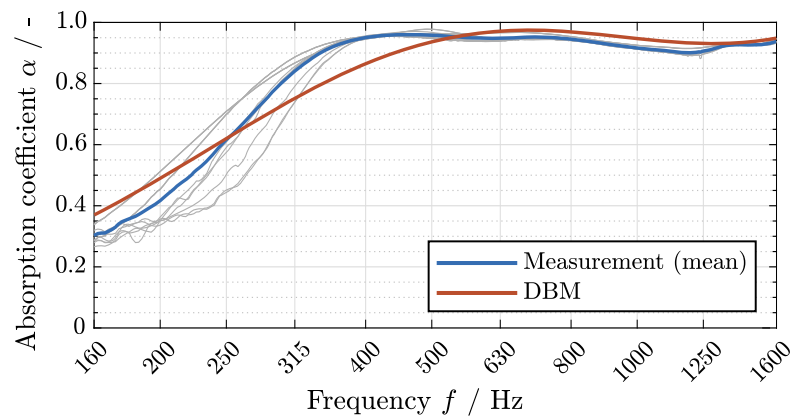

Fig. 8. Absorption curves for Baso Plan 100 obtained by measurement (grey curves, mean value shown in blue) and by the DBM model. (Color figure online)

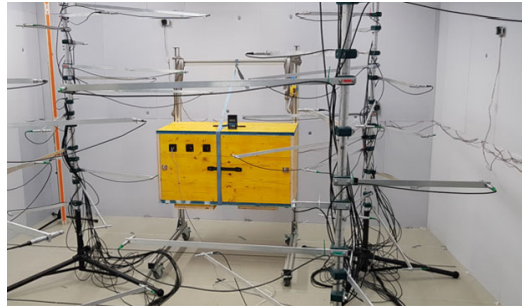

(a)

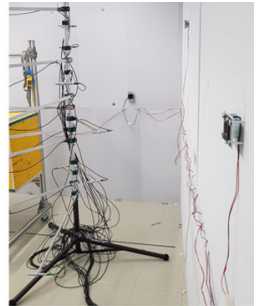

(b)
Fig. 9. Room with the generic sound source (yellow box) and the microphone trees as well as the mounted speakers on the wall. (Color figure online)

For the positioning within the room so-called microphone trees have been used (see Fig. 9). These trees can have different branches with different lengths at various heights to which the microphones are attached. For the application of the introduced inverse scheme the positions of these microphones have to be determined with respect to a reference position. For this purpose, an acoustic positioning system was developed [6], which is based on the principle of multi-lateration where the distances between an unknown position (microphone) and several known points (loudspeakers at the walls, see Fig. 9) is used to determine the microphone location. This setting is similar to the well-known global-positioning system (GPS) [39].

For the identification of the acoustic source, a microphone array with $M=50$ microphones is considered where the microphones are spatially distributed throughout the room without any special requirements for their positions. However, care was taken not to place them too close to the ceiling, the floor and the walls. The used microphone positions in the room, called MicMeas, are depicted in Fig. 10. Furthermore, an optimized arrangement named MicBest, which were obtained by simulations, was used for the inverse scheme (presented by black triangles). It has been shown that 2D microphone arrays do not provide satisfactory results for both beamforming and inverse schemes in the considered environment and therefore 2D arrays are not considered in this context (see [6]). 

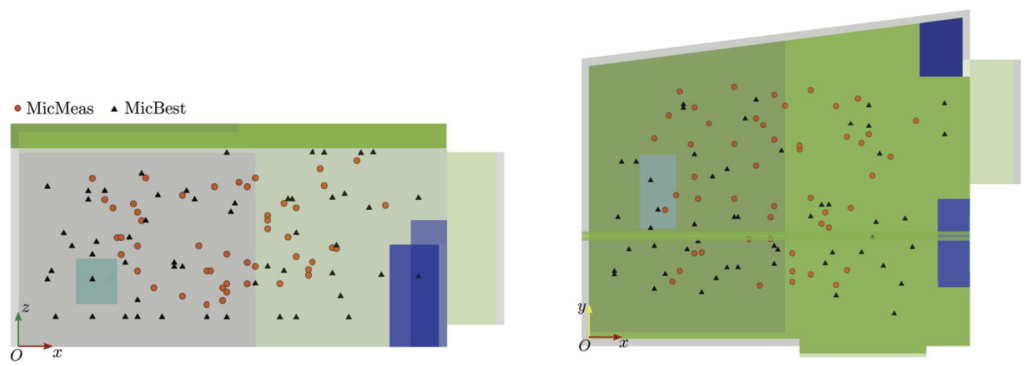

Fig. 10. Microphone locations in the considered measurement environment

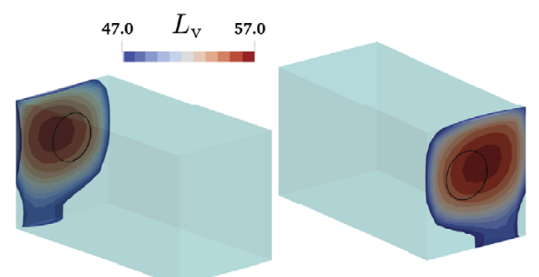

(a) Inverse Scheme.

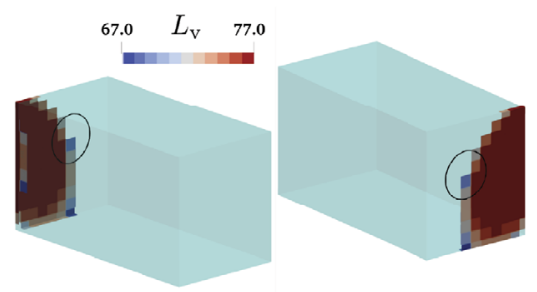

(c) FuncBF.

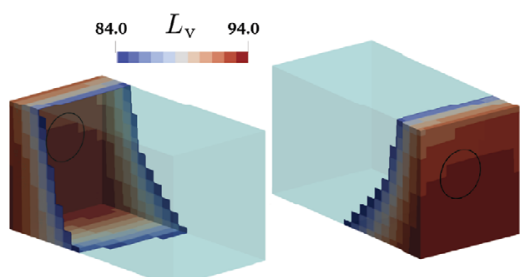

(b) ConvBF.

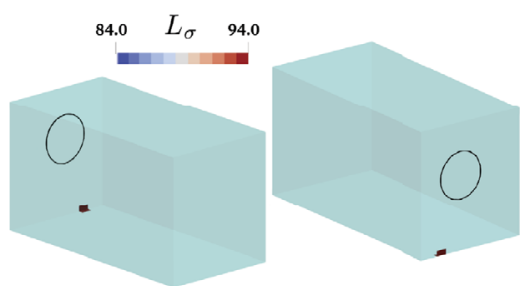

(d) Clean-SC.

Fig. 11. Result of the inverse scheme compared to acoustic beamforming algorithms (ConvBF, FuncBF, Clean-SC)

The temperature in the room was $\vartheta=25^{\circ} \pm 2^{\circ} \mathrm{C}$ and the relative humidity $\Phi=25 \pm 10 \%$ during the measurements. The considered source frequency is $250 \mathrm{~Hz}(\lambda \approx 1.36 \mathrm{~m})$. First, the localization result of speaker $\mathrm{L} 1$ on the left side of the generic source are shown in Fig. 11.

In addition to the inverse scheme, three common beamforming algorithms are used for the source identification to have a comparison with the results of the inverse scheme. Hereby, ConvBF and FuncBF were applied, which can also handle coherent acoustic sources. Furthermore, the Clean-SC deconvolution algorithm was applied. This algorithm removes the side lobes from the raw source map based on spatial source coherence. However, it will not work satisfactorily with several tonal (i.e. coherent) sound sources. The source maps are also shown in Fig. 11. Comparing the source maps of the inverse scheme with those of the beamforming algorithms used, we can see that the source map of the inverse scheme provides a more accurate localization.

So far, only the localization result has been considered, which indicates whether the source was identified at the correct position. In order to make quantitative statements about the identified source distribution, the identified sources will be used to perform a sound field computation. This allows comparisons with the original acoustic field measured at the microphone positions. For the inverse scheme this is straightforward and no further steps need to be taken, since a detailed source distribution both in amplitude and phase is identified. Hence, a numerical simulation was performed to obtain the acoustic field. For the quantification of the obtained result of the sound field computation, the relative L2 error

$$
p_{\mathrm{err}}=\sqrt{\frac{\sum_{m}^{M}\left(\widetilde{p}_{m}^{\text {inv }}-\widetilde{p}_{m}^{m s}\right)^{2}}{\sum_{m}^{M}\left(\widetilde{p}_{m}^{m s}\right)^{2}}}
$$

between measured $\widetilde{p}_{m}^{m s}$ and simulated pressure values $\widetilde{p}_{m}^{\text {inv }}$ at the microphone positions $M$ is used. The results are given in Table 1. For the beamforming source maps, the acoustic field computation is not as simple as for the source distributions obtained by the inverse scheme. Thereby, the main problem is given through the point spread function (PSF) of the array, since the computed source map (raw map) is a convolution of the PSF with the real source distribution. Deconvolution algorithms (like Clean-SC) try to eliminate the influence of the PSF from the raw source map resulting in a deconvolved map. Hence, the source maps obtained with Clean-SC can be integrated without further processing. For the ConvBF and FuncBF results, the source power integration technique [40] [41] [42] was applied to limit the effect of the PSF. Thereby, the raw source map is normalized by the integrated PSF for a point source in the center of the integration area. The integration needs a specified area. Thereby, the maximum of the source map was taken as the center point of the integration area. From this center point, a sphere with radius $0.1 \mathrm{~m}$ was assumed and all surface points in this sphere were used for the integration. The results in Table 1 demonstrate the main advantage of the inverse scheme, namely an accurate identification of the source distribution with amplitude and phase for the computation of the acoustic field. Hence, considering the source field 


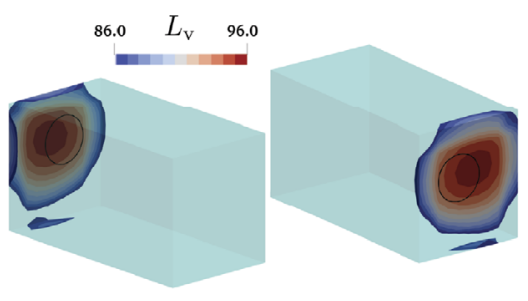

(a) Speaker L1

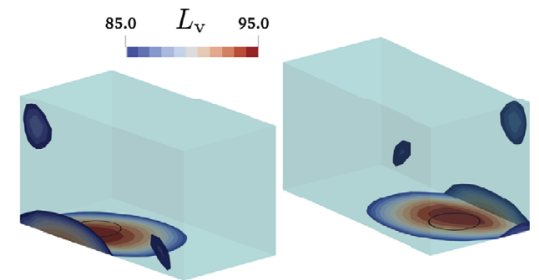

(b) Speaker L2

Fig. 12. Identified normal velocity level $L_{v}$

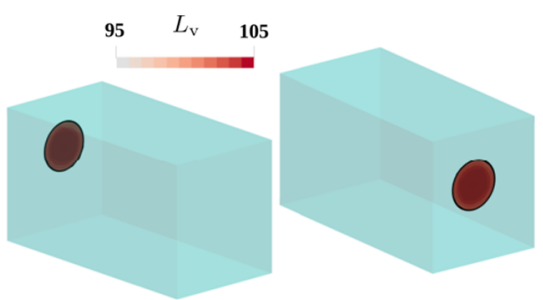

(a) True velocity level.

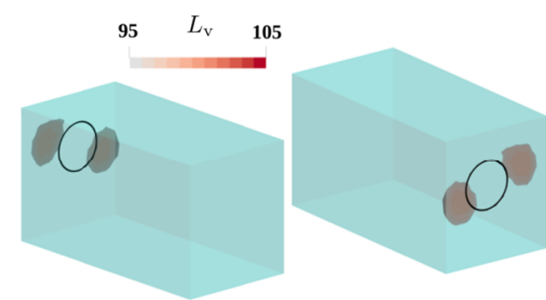

(b) MicMeas.

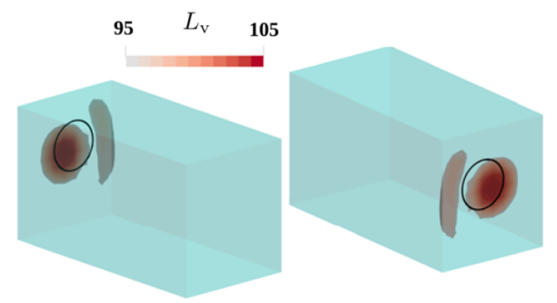

(c) MicBest.

Fig. 13. Identification results using (b)-(c) different microphone arrangements as well as the (a) true velocity level

Table 1. Error value $\boldsymbol{p}_{\mathrm{err}}$ in $\%$ using the identified sources depending on the microphone arrangement and the beamforming algorithm

\begin{tabular}{lllll}
\hline & ConvBF & FuncBF & Clean-SC & Inverse Scheme \\
\hline MicB & 107 & 84 & 107 & 20 \\
\hline
\end{tabular}

reconstruction, the inverse scheme clearly outperforms the beamforming methods.

Next, the identified normal velocity level $L_{v}$ by the inverse scheme is considered in order to have a comparison with the LSV measurement data (see Fig. 6). Herefore, first the same situation as before, when speaker L1 on the left side of the source is active, is considered (Fig. 12a). The direct comparison with the LSV data shows a deviation of about $17 \mathrm{~dB}$. In the next step, speaker $\mathrm{L} 2$ at the bottom of the box becomes the active sound source. This source radiates sound towards the floor. As before, the inverse scheme provides a good localization, but the amplitude again deviates by about $18 \mathrm{~dB}$ (Fig. 12b).

To demonstrate the capability of the inverse scheme by using highly sensitive microphone positions (named by MicBest, see Fig. 11), we proceeded as follows. A forward simulation with prescribed normal velocity at the loudspeaker position was performed (see Fig. 13a). The positions of the virtual microphones were determined using the guidelines in [6] such that location have been chosen, where the acoustic pressure has a maximum. Since the acoustic field is known in the room through the forward simulation, the positions can be found easily. Therefore, first the maximum in the sound pressure field was searched and taken as microphone position number one, whereby a constraint, namely that the microphone should not be too close to a reflective surface, is used. After this step, the position for the next microphone is determined. For this purpose, the region around the first microphone position is removed from the search space and the next maximum is searched. This procedure is followed until 50 positions are determined to have the same microphone number as before. In Fig. 13b,c the various identification results using the two arrangements are depicted. Thereby, MicB (microphone arrangement as used in the measurements) shows a good localization result with a deviation of the velocity level of about $10 \mathrm{~dB}$ compared to the original one. However, using the microphone arrangement MicBest an almost perfect localization result as well as a good agreement in velocity level could be achieved (see Fig. 13c).

The results achieved so far demonstrate the applicability of the proposed method for identifying low-frequency sound sources in real world situations. Thereby, an additional challenge is the localization (separation) of several active sound sources, especially in the low-frequency range. To test the proposed method in the presence of more than one acoustic source, two setups have been considered: (case A) speaker L1 and L3 active and (case B) speaker L1 and L2 active (case B). Both sources should have approximately the same source strength, since the excitation signal was the same for both. However, due to speaker tolerances, the same source level may not be achieved. By using the inverse scheme for localization a good identification especially for case A was achieved (see Fig. 14a). For case B (see Fig. 15a), the result is not as good, but we want to note that this setup is more challenging, since the two sources are 


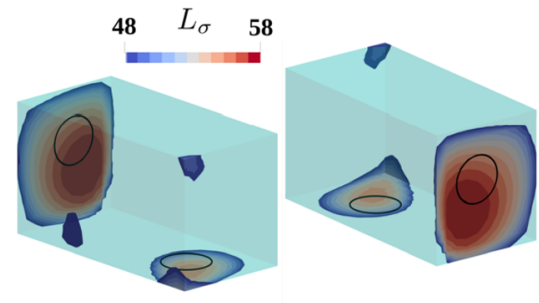

(a) Inverse scheme.

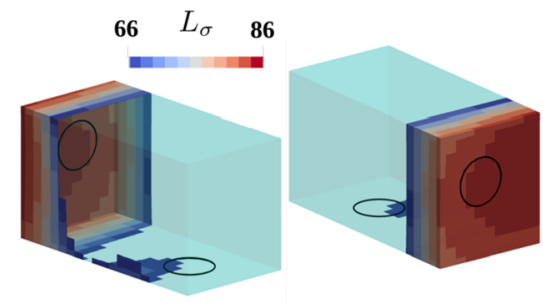

(b) FuncBF.

Fig. 14. Comparison of the source separation between inverse scheme and FuncBF (case A)

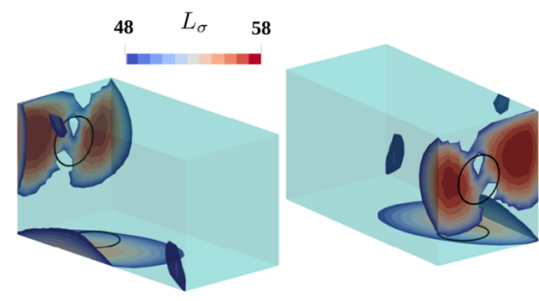

(a) Inverse scheme.

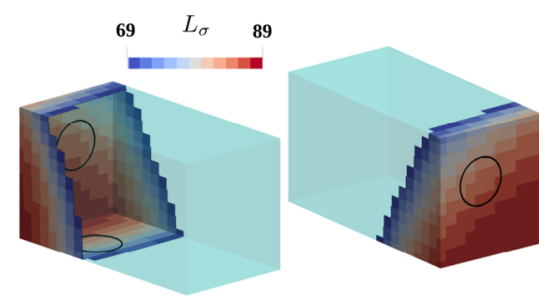

(b) FuncBF.

Fig. 15. Comparison of the source separation between inverse scheme and FuncBF (case B)

closer to each other which makes the separation harder. The localization was also done with FuncBF (ConvBF is omitted, because FuncBF has the better ability for source separation). Since CleanSC can not localize both sources (coherent sound sources), only the results of FuncBF will be considered. It can be observed that in case A (Fig. 14b), FuncBF can also separate the two sources, but the identified position of speaker L3 is more accurate with the inverse scheme. Moreover, the two source strengths of speaker L1 and $L 3$ (which should be approximately equal) do not differ as much. For case B, FuncBF can not separate between the two sources (see Fig. 15b).

\subsection{Rotating sources}

In the second application, measurement results of real world scenarios of rotating sound sources are presented [21]. All shown measurements were performed at FAU Erlangen with a ring array consisting of 64 microphones with a radius of $0.5 \mathrm{~m}$ [20]. For validation purposes, a fan with unskewed blades and mounted piezo buzzers was used. Next, measurements of a forward-skewed fan were processed. The radius of both fan blades is $0.25 \mathrm{~m}$.

Figure 16 shows results of ConvBF and Clean-SC of the unskewed fan with buzzers. The source maps depict the source level $L_{\sigma}$ defined in (6). The normal distance between array and fan plane is approximately $0.63 \mathrm{~m}$ and the rotational frequency of the fan $590 \mathrm{~min}^{-1}$. The frequency band of the scanning frequency $f_{\text {scan }}$ was chosen $2 \mathrm{kHz} \leq f_{\text {scan }} \leq 6 \mathrm{kHz}$ which is the frequency range of the buzzers. The averaging of the source strengths computed at single frequencies within the defined band was performed according to (5). The resulting source maps provide an acoustic image of the position the fan had when the measurement was started. In order to interpret the locations of the sound sources with respect to the fan geometry correctly, it is necessary to measure the angle $\varphi(t)$ of the fan synchronously with the emitted sound pressures.

As can be seen, there is no significant difference between interpolation method and spinningmode decomposition presented in Sect. 2.4 regarding the source maps, i.e. positions and levels of the identified sources. If no signal processing was performed prior to the beamforming algorithm, thus stationary sources were assumed, the source maps would be smeared and the piezo buzzers would be interpreted as ring shaped sources. Figures $16 \mathrm{a}$ and $16 \mathrm{~b}$ show results of ConvBF, which show distinctive peaks (main lobes) where the sources are located. There can be seen three main sources near three of the blade tips where the buzzers are mounted. Due to the frequencies of the sound sources and the geometry of the used array and setup, the spacial resolution is not high enough to determine if there is only one buzzer mounted on a blade or more close to each other. Further, there are side lobes of all three main sources visible that interfere with each other.

Figures $16 \mathrm{c}$ and $16 \mathrm{~d}$ show results of Clean-SC which is a deconvolution algorithm that uses the source maps calculated with ConvBF, also called "dirty maps", as basis. As this method removes the side lobes of the ConvBF-map and incoherent sources are replaced by single peaks, the individual buzzers at each blade show as separate sound sources in the map. The sources on the fan blade located in the first quadrant now can be clearly identified as independent sources, whereas in case of ConvBF the amplitude of their main lobe is in the range of the amplitudes of the side lobes and therefore the source position could be mistaken as an artefact. Again, there is very good agreement between interpolation method and spinningmode decomposition.

Figure 17 shows source maps of the forward-skewed fan with same radii of array and fan, respectively. The distance between array and fan in this setup is approximaletly $0.71 \mathrm{~m}$, the rotational frequency is $1486 \mathrm{~min}^{-1}$ and the scanning frequency is chosen $3 \mathrm{kHz} \leq f_{\text {scan }} \leq 4 \mathrm{kHz}$. Here, no additional sources as in the validation setup with piezo buzzers are mounted. Therefore the overall level of the source level is lower. The fan has nine blades and the Clean-SC algorithm identifies several sources along each blade. Again, there is good agreement between the two methods concerning locations and amplitudes of the sources. 


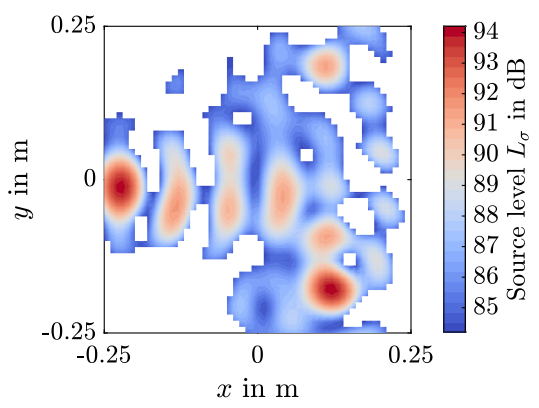

(a) ConvBF, Spinningmode decomposition.

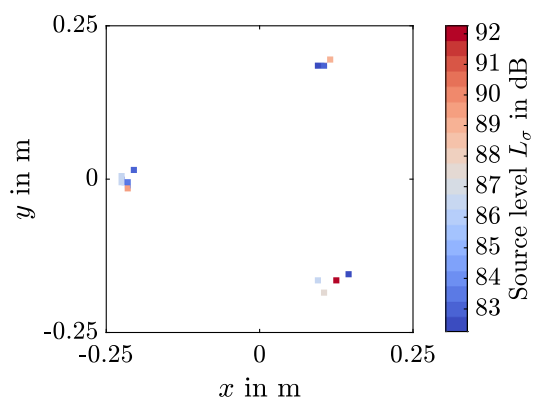

(c) Clean-SC, Spinningmode decomposition.

Fig. 16. Source maps of a fan with piezo buzzers, $2 \mathrm{kHz} \leq f_{\text {scan }} \leq 6 \mathrm{kHz}$

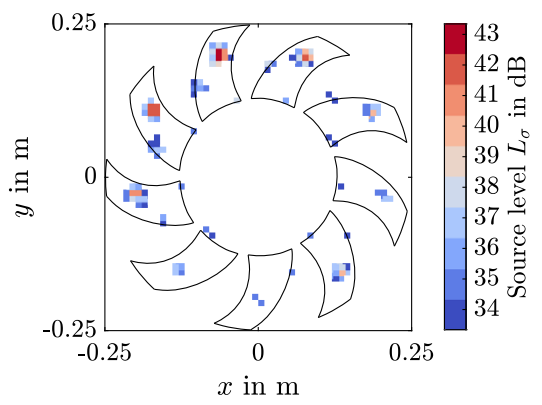

(a) Spinningmode decomposition

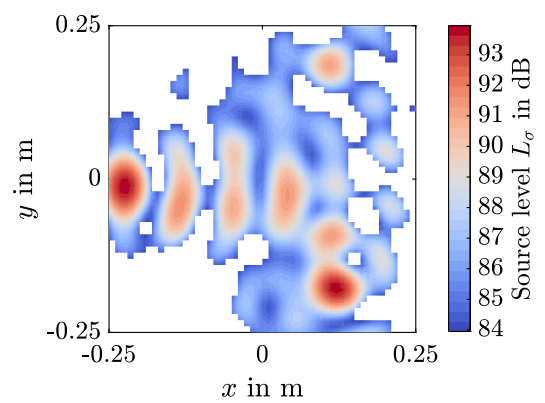

(b) ConvBF, Interpolation method.

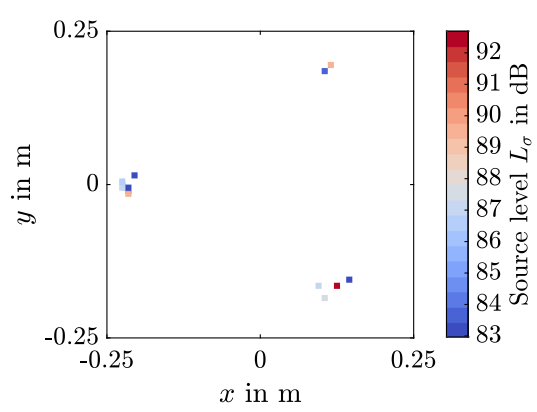

(d) Clean-SC, Interpolation method.

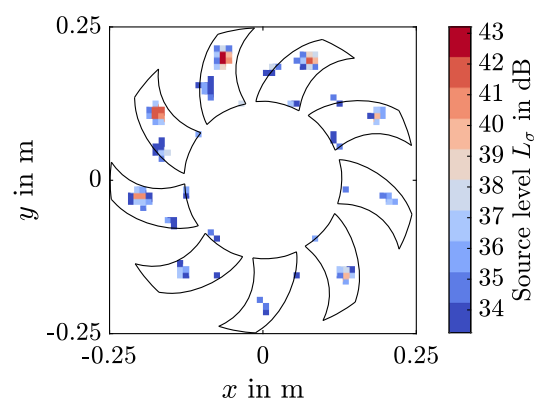

(b) Interpolation method.

Fig. 17. Source maps of forward-skewed fan, Clean-SC, $3 \mathrm{kHz} \leq f_{\mathrm{scan}} \leq 4 \mathrm{kHz}$

4.2.1 Results for different steering vectors

As mentioned in Sect. 2.4, finding the correct source positions of rotating sources requires not only signal processing of the recorded pressure signals but also the use of the correct Green's function $\widetilde{g}_{\Omega}$ for rotating monopoles as steering vector. Using $\widetilde{g}_{\Omega}$ and the spinningmode decomposition is the only analytically exact method. However, the Green's function $\widetilde{g}_{0}$ for stationary monopoles can be used as an approximation to $\widetilde{g}_{\Omega}$. Additionally, corrected distances between each assumed source position on the source grid and each microphone can be calculated and inserted into the stationary Green's function. The function $\widetilde{g}_{0}\left(r_{m}^{*}\right)$ with corrected distances $r_{m}^{*}$ can be used as an improved approximation to $\widetilde{g}_{\Omega}$.

In Fig. 18 all three options are depicted. For these figures measurement data of the unskewed fan with buzzers is used but with a scanning frequency $f_{\text {scan }}$ between 4 and $6 \mathrm{kHz}$ so that only the buzzers on one blade are visible. The actual source position is marked with a black circle. If the rough approximation $\widetilde{g}_{\Omega} \approx \widetilde{g}_{0}(r)$ is used (Fig. 18a), the identified source position is shifted in $\varphi$-direction (tangential direction) and the source map gets somewhat smeared. In case of the use of $\widetilde{g}_{0}\left(r_{m}^{*}\right)$ (Fig. 18b) or $\widetilde{g}_{\Omega}$ (Fig. 18c) the correct source position is identified. The scale of deviation from the real source position depends on the rotational frequency and the distances between source grid and observer positions.

As the calculation of the correct Green's function $\widetilde{g}_{\Omega}$ requires, amongst others, evaluation of spherical harmonics and therefore comes at high computational costs, the approximation with $\widetilde{g}_{0}\left(r_{m}^{*}\right)$ is a good trade-off between accuracy and computational time, as the corrected distances are calculated once for a measurement setup for each possible combination of scanning point and microphone position but they are not frequency-dependable.

\section{Conclusion}

In this contribution an overview of beamforming based algorithms for sound source localization was presented and the advantages as 


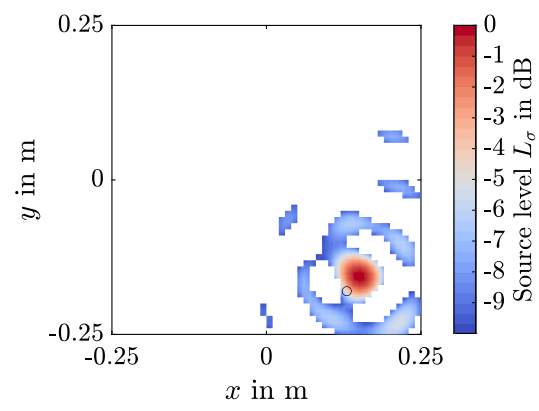

(a) Spinningmode decomposition, $\tilde{g}=$ $\tilde{g}_{0}(r)$

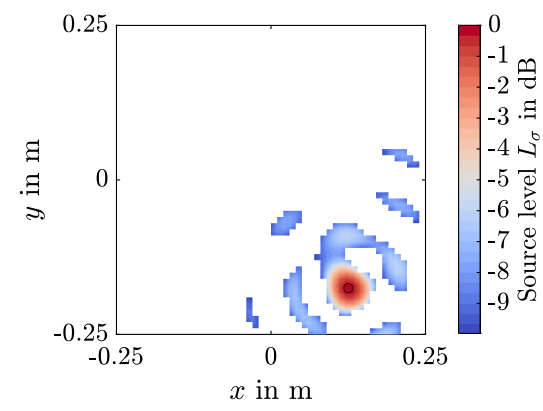

(b) Spinningmode decomposition, $\tilde{g}=$ $\tilde{g}_{0}\left(r_{m}^{*}\right)$

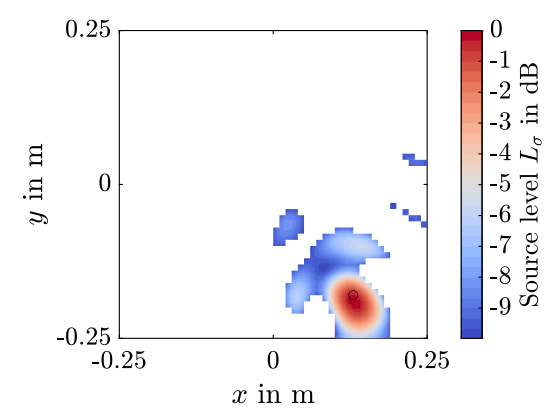

(c) Spinningmode decomposition, $\tilde{g}=$ $\tilde{g}_{\Omega}(r)$

Fig. 18. Source maps of a fan with piezo buzzers with different steering vectors, ConvBF, $4 \mathrm{kHz} \leq f_{\mathrm{scan}} \leq 6 \mathrm{kHz} \circ \ldots$ actual source position

well as limitations of the different algorithms were discussed. Further, two methods for source localization for rotating sound sources are presented. For each method, real-world microphone measurements were performed and evaluated.

A main restriction in current beamforming methods is given by the assumption of free radiation for the calculation of the transfer function between microphone and assumed source point (steering vector). This transfer function is usually given by Green's function for free radiation. Hence, obstacles as well as absorbing surfaces are not considerable. In the presented inverse scheme the Helmholtz equation with the correct boundary conditions is solved. Thereby, to recover the source locations, an inverse scheme based on a sparsity promoting Tikhonov functional to match measured (microphone signals) and simulated pressure is used. A clear advantage of such an inverse method is its ability to fully consider realistic geometry and boundary condition scenarios, as well as its straightforward generalizability to situations with convection and/or damping. Furthermore, a detailed source distribution both in amplitude and phase is identified, and finally with these information a numerical simulation can be performed to obtain the acoustic field. Standard beamforming methods require an integration (question of the integration area arises) or more complex convolution algorithms must be applied.

The localization results achieved with the inverse scheme demonstrates the applicability at low-frequencies in real-world scenarios. Furthermore, through simulations, it could be shown that a perfect reconstruction result of the acoustic sources can be achieved with microphone positions at pressure maxima, which demonstrates the potential of the inverse scheme. Despite the superiority of the inverse scheme compared to advanced deconvolution based signal processing schemes one has to consider the high effort based on the two challenges: (1) Geometry and boundary condition modeling for an accurate FE computation, and (2) the precise determination of the microphone positions.

Considering beamforming at rotating sound sources, it could be shown that both presented methods, the interpolation in time domain and spinningmode decomposition in frequency domain, provide good results.

Funding Note Open access funding provided by TU Wien (TUW).

Publisher's Note Springer Nature remains neutral with regard to jurisdictional claims in published maps and institutional affiliations.

Open Access Dieser Artikel wird unter der Creative Commons Namensnennung 4.0 International Lizenz veröffentlicht, welche die Nutzung, Vervielfältigung, Bearbeitung, Verbreitung und Wiedergabe in jeglichem Medium und Format erlaubt, sofern Sie den/die ursprünglichen Autor(en) und die Quelle ordnungsgemäß nennen, einen Link zur Creative Commons Lizenz beifügen und angeben, ob Änderungen vorgenommen wurden. Die in diesem Artikel enthaltenen Bilder und sonstiges Drittmaterial unterliegen ebenfalls der genannten Creative Commons Lizenz, sofern sich aus der Abbildungslegende nichts anderes ergibt. Sofern das betreffende Material nicht unter der genannten Creative Commons Lizenz steht und die betreffende Handlung nicht nach gesetzlichen Vorschriften erlaubt ist, ist für die oben aufgeführten Weiterverwendungen des Materials die Einwilligung des jeweiligen Rechteinhabers einzuholen. Weitere Details zur Lizenz entnehmen Sie bitte der Lizenzinformation auf http://creativecommons.org/licenses/by/4.0/ deed.de.

\section{References}

1. Mueller, T. J. (2002): Aeroacoustic measurements. In Beamforming in Acoustic Testing (S. 62-97). Berlin: Springer.

2. Herold, G., Sarradj, E. (2017): Performance analysis of microphone array methods. J. Sound Vib., 401, 152-168.

3. Fischer, J., Doolan, C. (2017): Beamforming in a reverberant environment using numerical and experimental steering vector formulations. Mech. Syst. Signal Process., $91,10-22$. 
4. Sarradj, E. (2012): Three-dimensional acoustic sourcemapping with different beamforming steering vector formulations. Adv. Acoust. Vib., 2012, 292695.

5. Gombots, S., Kaltenbacher, M., Kaltenbacher, B. (2016): Combined ExperimentalSimulation Based Acoustic Source Localization, Fortschritte der Akustik. DAGA 2016. Deut. Jahrestagung Akust., 42, 1092-1095.

6. Gombots, S. (2020): Acoustic source localization at low frequencies using microphone arrays. PhD Thesis, TU Wien.

7. Dougherty, R., Walker, B. (2009): Virtual Rotating Microphone Imaging of Broadband Fan Noise, 15th AIAA/CEAS Aeroacoustics Conference (30th AIAA Aeroacoustics Conference). AIAA 2009-3121.

8. Johnson, D. H., Dudgeon, D. E. (1993): Array signal processing: concepts and techniques. In Finite Continuous Apertures (S. 64-65). Englewood Cliffs: PTR Prentice Hall.

9. Dougherty, R. P., Ramachandran, R. C., Raman, G. (2013): Deconvolution of sources in aeroacoustic images from phased microphone arrays using linear programming. Int. J. Aeroacoust., 12(7-8), 699-717.

10. Dougherty, R. P. (2014): Functional Beamforming. In 5th Berlin Beamforming Conference. BeBeC-2014-01.

11. Dougherty, R. P. (2014): Functional Beamforming for Aeroacoustic Source Distributions, 20th AIAA/CEAS Aeroacoustics Conference. AIAA 2014-3066.

12. Brooks, T. F., Humphreys, W. M. (2006): A deconvolution approach for the mapping of acoustic sources (damas) determined from phased microphone arrays. J. Sound Vib., 294, 856-879.

13. Sijtsma, P. (2007): CLEAN based on spatial source coherence. Int. J. Aeroacoust., 6(4), 357-374.

14. Yardibi, T., Li, J., Stoica, P., Cattafesta, L. N. (2008): Sparsity constrained deconvolution approaches for acoustic source mapping. J. Acoust. Soc. Am., 123(5), 2631-2642.

15. Chu, Z., Yang, Y. (2014): Comparison of deconvolution methods for the visualization of acoustic sources based on cross-spectral imaging function beamforming. Mech. Syst. Signal Poces., 48(3), 404-422.

16. Padois, T., Berry, A. (2017): Two and Three-Dimensional Sound Source Localization with Beamforming and Several Deconvolution Techniques. Acta Acust. Acust., 103(3), 357-392.

17. Dowling, A. P., Ffowcs, W. J. (1983): Sound and sources of sound. Chichester: E. Horwood. 1983.

18. Herold, G., Sarradj, E. (2015): Microphone array method for the characterization of rotating sound sources in axial fans. Noise Control Eng. J., 63, 546-551.

19. Dougherty, R., Walker, B. (2009): Virtual Rotating Microphone Imaging of Broadband Fan Noise., 15th AIAA/CEAS Aeroacoustics Conference (30th AIAA Aeroacoustics Conference). Aeroacoustics Conferences. Washington: AIAA

20. Krömer, F. (2018): Sound emission of low-pressure axial fans under distorted inflow conditions, FAU Forschungen, Reihe B, Medizin. Naturwissenschaft, Technik.

21. Nowak, J., Krömer, F., Kaltenbacher, M. (2019): Vergleich verschiedener Methoden zur Schallquellenlokalisation bei Axialventilatoren, Fortschritte der Akustik. DAGA 2019. Deut. Jahrestagung Akust., 45, 31-34. 2019.

22. Sarradj, E. (2008): Quantitative source spectra from acoustic array measurements. In 2nd Berlin Beamforming Conference. BeBeC-2008-03.

23. Sarradj, E., Geyer, T., Brick, H., Kirchner, K.-R., Kohrs, T. (2012): In Application of Beamforming and Deconvolution Techniques to Aeroacoustic Sources at Highspeed Trains, NOVEM - Noise and Vibration: Emerging Methods, Sorrento.

\section{Authors}

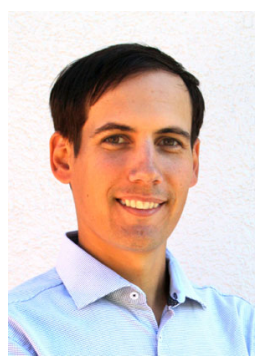

\section{Stefan Gombots}

graduated in Mechanical Engineering from TU Wien in 2015 and received his PhD from TU Wien in 2020. His research focuses on sound source localization utilizing acoustic measurements and simulations.
24. Merino-Martínez, R., Sijtsma, P., Carpio, A. R.,Zamponi, R., Luesutthiviboon, S., Malgoezar, A. M. N., Snellen, M., Schram, C., Simons, D. G. (2019): Integration methods for distributed sound sources. Int. J. Aeroacoust., 18(4-5), 444-469.

25. Leclere, Q., Pereira, A., Bailly, C., Antoni, J., Picard, C. (2017): A unified formalism for acoustic imaging based on microphone array measurements. Int. J. Aeroacoust., 16 431-456.

26. Ehrenfried, K., Koop, L. (2007): Comparison of Iterative Deconvolution Algorithms for the Mapping of Acoustic Sources. AIAA J., 45(7), 1584-1595.

27. Yardibi, T., Zawodny, N. S., Bahr, C., Liu, F., Cattafesta, L. N., Li, J. (2010): Comparison of Microphone Array Processing Techniques for Aeroacoustic Measurements. Int. J. Aeroacoust., 9(6), 733-761.

28. Merino-Martínez, R., Sijtsma, P., Snellen, M., Ahlefeldt, T., Bahr, C. J., Blacodon, D., Ernst, D., Finez, A., Funke, S., Geyer, T. F., Haxter, S., Herold, G., Huang, X., Humphreys WIIlam, W. M., Leclère, Q., Malgoezar, A., Michel, U., Padois, T., Pereira, A., Picard, C., Sarradj, E., Siller, H., Simons, D. G., Spehr, C. (2019): A Review of Acoustic Imaging Methods Using Phased Microphone Arrays. CEAS Aeronaut. J., 10, 197-230.

29. Chiariotti, P., Martarelli, M., Castellini, P. (2019): Acoustic beamforming for noise source localization: Reviews, methodology and applications. Mech. Syst. Signal Process., 120, 422-448.

30. Suzuki, T. (2011): L1 generalized inverse beam-forming algorithm resolving coherent/incoherent, distributed and multipole sources. J. Sound Vib., 330, 5835-5851.

31. Deckers, E, Jonckheere, S., Vandepitte, D. Desmet, W. (2015): Modelling techniques for vibro-acoustic dynamics of poroelastic materials. Arch. Comput. Methods Eng., 22(2), 183-236.

32. Anzengruber, S. W., Hofmann, B., Mathé, P. (2014): Regularization properties of the sequential discrepancy principle for Tikhonov regularization in Banach spaces. Appl. Anal., 93(7), 1382-1400.

33. Lu, S., Pereverzev, S. V. (2011): Multi-parameter regularization and its numerical realization. Numer. Math., 118(1), 1-31.

34. Kaltenbacher, M., Kaltenbacher, B., Gombots, S. (2018): Inverse Scheme for Acoustic Source Localization using Microphone Measurements and Finite Element Simulations. Acta Acust. Acust., 104, 647-656.

35. Kaltenbacher, M. (2015): Numerical Simulation of Mechatronic Sensors and Actuators: Finite Elements for Computational Multiphysics. 3. ed. Berlin: Springer.

36. ISO 10534-2 (2001): Acoustics - Determination of sound absorption coefficient and impedance in impedance tubes - Part 2: Transfer-function method.

37. Delany, M. E., Bazley, E. N. (1970): Acoustical properties of fibrous absorbent materials. Appl. Acoust., 3(2), 105-116.

38. Delany, M. E., Bazley, E. N. (1990): Acoustical properties of porous materials. Modifications of Delany-Bazley models. J. Acoust. Soc. Jpn., 11(1), 19-24.

39. Hofmann-Wellenhof, B., Lichtenegger, H., Collins, J. (2012): Global positioning system: theory and practice. Berlin: Springer.

40. Brooks, T. F., Humphreys, W. M. (1999): Effect of directional array size on the measurement of airframe noise components. In 5 th AIAA/CEAS Aeroacoustics Conference and Exhibit (S. 99-1958). Washington: AIAA.

41. Sijtsma, P. (2010): Phased Array Beamforming Applied to Wind Tunnel And Fly-Ove Tests. SAE Brasil International Noise and Vibration Congress. SAE International.

42. Merino-Martínez, R., Neri, E., Snellen, M., Kennedy, J., Simons, D., Bennett, G. J. (2017): Comparing flyover noise measurements to full-scale nose landing gear wind tunnel experiments for regional aircraft. In 23rd AIAA/CEAS Aeroacoustics Conference. AIAA 2017-3006.

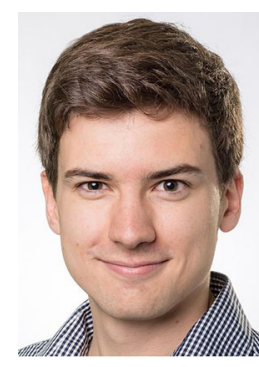

\section{Jonathan Nowak}

graduated from TU Wien in 2018 with a masters degree in Mechanical Engineering - Economics. The topic of his master thesis was sound source localization of rotating sources. Since 2018 he has been working under the supervision of Prof. Manfred Kaltenbacher as a university assistant at the Institute of Mechanics and Mechatronics at TU Wien in the research unit of Technical Acoustics. His main research topic is sound source localization using microphone measurements and the Finite Element method. His teaching responsibilities invlove the exam and exercise of Measurement and Vibration Technology. 


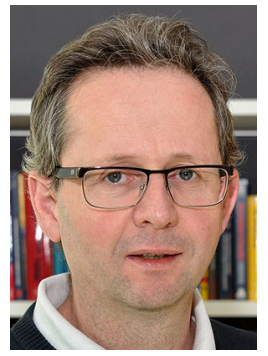

Manfred Kaltenbacher

received his Dipl.-Ing. in electrical engineering from the Technical University of Graz, Austria in 1992, his Ph.D. in technical science from the Johannes Kepler University of Linz, Austria in 1996, and his habilitation from Friedrich-Alexander-University of ErlangenNuremberg, Germany, in 2004. In 2008 he became a full professor for Applied Mechatronics at Alps-Adriatic University Klagenfurt,
Austria. In 2012 he moved to TU Wien, Austria, as a full professor for Measurement and Actuator Technology, and in 2020 he became the head of the Institute of Fundamentals and Theory in Electrical Engineering at TU Graz, Austria. His research involves theory, modeling, simulation and experimental investigation of complex systems in engineering, material and medical science. A main focus is on the development of advanced Finite Element (FE) methods for multi-field problems (electromagnetics-mechanics, mechanics-acoustics, piezoelectricity, flow dynamics - mechanics, aeroacoustics), and their application to design mechatronic sensors and actuators. 